\title{
Urban Encounters Reloaded: towards a descriptive account of augmented space
}

(Please do not include author information for an initial submission to ensure a double blind review process)

\author{
Timothy Jung ${ }^{\mathrm{a}}$, \\ Name of $2^{\text {nd }}$ author ${ }^{\mathrm{a}}$, and \\ Name of $3^{\text {rd }}$ author - different University (no titles, affiliations etc.) ${ }^{\mathrm{b}}$ \\ ${ }^{\text {a }}$ Faculty of Business and Law \\ Manchester Metropolitan University, United Kingdom \\ t.jung@mmu.ac.uk \\ ${ }^{\mathrm{b}}$ Name of the institute / department \\ Name of the University, Country \\ author3@email.edu
}

\begin{abstract}
In this chapter, augmented space is described as the layering of media technologies onto the physical space of the city. The approach assesses salient aspects of the experience of space in everyday life, the city and urban space more generally. The chapter discusses these in relation to the deployment of augmenting technologies and modes of display associated with augmented reality, new and digital media: visual or otherwise. Selected work, carried out in relation to culture, leisure and tourism is assessed. These case studies indicate the potential of augmented reality in areas of a) urban design, b) tourism and heritage, and c) the promotion of cycling for health and the creation of alternative transport infrastructure. The main characteristics of AR and augmented space are presented. This is followed by a discussion and development of hybrid research tools and applied in two case studies with a view to providing a potential roadmap for future work in this area.
\end{abstract}

Keywords: augmented reality, augmented space, ubiquitous and mobile technologies, human engagement, media layer, site-specific, embodiment, heritage and tourism, cycling and sustainable transport

\section{Introduction}

Our urban encounters with the digital such as those reported in Fatah (2008), Allen (2008) and Robison (2012) have gained in their in urgency largely as a consequence of the popularising of 'real world' augmented reality applications in gaming such as Ingress and more recently Pokemon Go (Humphery-Jenner, 2016). These application have emphasised the potential for the gamification of real spaces and, like geocacheing and other activities have moved computer gameplay from the console or desktop into the open air. As suggested by its title, the overriding intention of this chapter is to update our current knowledge of urban space and the experience of the city that we argue has come about as a consequence of these new developments. Through the lens of the selected work, we will examine the potential integration of augmented reality into real space as well as some of the consequences of this. 


\section{The strange case of Pokemon Go!}

The arrival of Pokemon Go in July 2116 was a 'wake up call' for anyone engaged in academic research into augmented reality, in fact, it was a wake up call for anyone in the AR industry. It's immediate popularity overcame all expectations and whilst the hype died down surprisingly quickly, putting one in mind of Gartner's "hype cycle" (Gartner, 2016) the sudden spike in interest did, however, give Nintendo a much needed opportunity to reawaken its sales in merchandising. Niantic had already had gamers explore the open air with Ingress since November 2012. Pokemon Go's lack of financial sustainability within this new potential market for augmented reality gaming was striking: "By mid-September, daily revenues had fallen from US\$16m per day to US $\$ 2 \mathrm{~m}$ (excluding the $30 \%$ app store fee) and daily downloads had declined from a peak of 27 million to 700,000" Humphery-Jenner, 2016). The platform raises interesting safety and risk perception issues. These issues may arise in the need for appropriate warnings about gamer proximity to nearby hazards or, indeed, other game players. Attempts to rectify these are evident in the recent release of Pokemon Go Plus, a small piece of hardware that apparently performs the necessary proximity detection to keep the gamer safe. But Tassi (2016) writes that this comes with a significant loss of gameplay and user engagement.

In terms of academic research, the Pokemon Go phenomenon raises some tantalizing issues:

- The use of augmented reality in gaming (and the problems associated with this).

- The move of computer games and entertainment media from the desktop and console into the open air.

- The key importance of gamer location and attachment to place through locative technologies such as GPS.

- The 'whole deal' around the use and design of space into which AR is introduced.

- The changing of the experience of space to experience of place, whether participants are 'being-in' or 'being-out' of real space and the observation, or otherwise, of spatial constraints and social norms.

- The dynamic relations between the real and the virtual within AR systems.

- The pressing need to consider the body and its movements in public space, as a key component within augmented reality systems.

- Aspects of engagement and gamification in public space through the use of rewards and similar motivating objects.

At the very least, new standards for delivery and new research methods are required. In what follows, we propose a potential research methodology and an overall argument intended to facilitate a much greater understanding of how augmented reality can be incorporated into real world applications and real spaces, but with awareness of the pitfalls and risk perception variables associated with the introduction of Pokemon Go and other 'AR in the wild' (Rogers, 2011) applications. 
Theoretical and conceptual work about augmented space have tended to be presented from the perspective of the generalised application of a wider range of ubiquitous and mobile technologies applied to the experience of the city, as in Manovich's Poetics (2006). Many urban spaces are already augmented with a wide range of ubiquitous and mobile technologies, in addition to other forms of media display, including urban screens, for example and as such can easily be described as "augmented spaces" (Aurigi 2008).

These accounts (Aurigi 2008, Allen 2008 and Fatah 2008), give priority to the spatial aspects of the design of these spaces and how any intervention, whether as small scale displays using a handheld device or as large scale media displays, can be integrated into the environment in which it is situated (Allen 2008 and Fatah 2006). The debate is about this general shift towards augmentation and the human experience of urban and public space being transformed or disrupted by its introduction. The extent to which this can be seen as transnational and generalized phenomenon is also worth discussion.

The brief case study descriptions later in this chapter have the purpose of developing a theoretical understanding of augmented space alongside discussion of the practical development of mobile apps. We argue that it is necessary to go beyond our current understanding of human-computer interaction and related methodologies to understand technology use in the context of augmented space. User participation and engagement is a key concern because the existing strategies used for empirical work in this area may not be enough to cope with both the complexity of human engagement as well as the global nature of these and other related media.

The design, development and deployment of augmented reality systems and their novel forms of display can be evaluated in terms of their facility to create, develop and promote to particular target groups and audiences. That is to say, the work presented in this chapter indicates the development of alternative research, ones that are not necessarily new but that are being applied in different ways to new contexts and combined into a transdisciplinary research strategy. The methods described in this chapter are being devised in order to shed some light on both participation and engagement in the following areas: audience development for museums and galleries and other similar forms of cultural space outdoors, the promotion of cycling, the development of sustainable transport infrastructure and the promotion of healthy lifestyles. These aspects of our empirical strategy are useful additions to our existing research into the design and use of urban and public space.

\section{Some Characteristics of Augmented Space}

This section provides a descriptive account of some of the characteristics that are considered critical to the existence of augmented space. As such, it is an account that avoids a straightforward definition of augmented space. Similar to the account of "characteristics of augmented reality" provided by Azuma (1997), where the survey given there avoided a full technical description of AR. Indeed, given the variety of 
technical systems now used in the development of AR, such an account would be so complex, and, given the general perspective of augmented space provided in this chapter, such a definition might be rendered meaningless. Characteristics that are of importance to the work presented here are similar to Azumi's previous account of augmented reality and include: the combination of the real and the virtual, interaction and presentation in real time, and frequent registering in three dimensions.

As was indicated in the previous section a distinction is made between augmented reality and augmented space. The former of these gives priority to the architectural, urban and lived in space within which any augmentation takes place. Previous research on augmented space has tended to focus on the impact of a range of media technologies onto the experience of urban space and put in the context of the layering of these technologies (see e.g. Allen 2008, 2009 and Robison 2012) and how they are superimposed onto the built environment including work on analysing patterns of use associated with the BBC's Big Screen network in the UK and the use of mobile gaming as an encouragement tool for social interaction and language learning when travelling to locations of cultural and historical interest.

\subsection{The site-specific nature of augmented space}

Salient observations that have been generated from a consideration of the characteristics of augmented space in the urban context have been developed over many years and stem largely from an interest in the widespread use of large scale LCD displays into the built environment. Here it has already been established that there is a site-specific and located quality to the medium. It has been argued (Allen 2012) that there is a "site-specific" (Kwon 2004) quality to the manifestation of these screens.

McCarthy argued this in relation to the occurrence of smaller scale video displays and TVs in everyday settings such as shopping malls, in bars etc. (McCarthy 2003). The concept of site specificity was then applied to large scale urban screens and it was established that there was quite a powerful located aspect to both the positioning of the screens and the type of content that was displayed on them, as well as important specifics of the visual design of content (Allen 2008, 2009). Case studies on the screen in city centre of Bradford (ibid), for example, showed important characteristic of augmented space more generally and here it is argued, also becomes an important characteristic of 'applied' augmented in an urban setting. In that instance, the physical location of the screens was key to understanding their function and use within the city centre; their evolution and institutional underpinnings (funding, management and curatorial practices); variability of the local environment; simple physical features such as their height; their proximity to particular shops and spaces - in the case of Bradford's screen, adjacent to a major photography gallery. Anna McCarthy (2003, p. 197) has argued that the relation between television and its integration into public space, into sites such as cafes, shops, information displays, travel interchanges, into a wide variety of public areas and into the built environment in more general, is a site specific relation. 
Much research has already taken place in relation to the use of "pervasive technology" and its relations to the design and use of space in the urban context (Fatah, 2008) and, more importantly, the new forms of interaction that these "digital flows" can facilitate. All of the research indicated here has attempted to integrate an interest in the use of a range of display technologies, whether large or small scale, with an interest in understanding salient aspects of the design of the built environment and the way that they are used by inhabitants of the city. In this section, therefore, the overriding question is whether AR can be placed within the wider context of the debate concerning augmented space. Is AR destined to become yet another layer to add to an already complex set of technologies and systems that persistently confront inhabitants of the city? This style of approach has already been applied to forms of augmented space through the application of Space Syntax as methodology. "Space Syntax analyses cities as systems of space created by physical artefacts of architecture and urban design" (Fatah, 2008, p. 4). The tendency in this research is to focus on specific spatial characteristics of a given location and how those digital flows impact upon the space and forms of interaction. This is precisely the methodology to be used in order to understand the use of AR in urban space.

This focus on space and the deployment of pervasive systems into spaces in the city has emphasised a very important relation and this is proximity and the spatial relations that are established between devices and, therefore, between users of the technology, as well as other objects that might be present within the environment that can be detected digitally. "Knowing people's 'Bluetooth trails' can help us identify the direction of the movement of a particular device". Giving rise to a potential understanding of, so called "digital attractors" (Fatah, 2008, p. 11).

Proxemic relation as indicated in the above can be reflected in a representational and semiotic context in the sense that the closer that an element is in spatial terms to another, say if this were to people in proximity both holding devices, the likelier they are to be seen as in a relation. They are connected in some way purely by their proximity to one another. It is here that the spatial properties of both the medium but also the surroundings within which consumption or reception takes place and both are bound up with forms of social action. This notion of proxemics, first developed by Hall (1966) and later applied to social semiotics and the multimodal properties of texts, Hodge and Kress (1998) and has found more recent articulation in the analysis of urban space, O'Toole (2001), Alias (2004) and our own work. The application of proxemics and proxemic coding might prove to be fruitful to the integration of augmented reality to urban space in the sense that there are social meanings, perceptions and consequences that are generated or triggered by proximity. Indeed, when applied to some of the spatial and interactive issues associated with Pokemon Go it is noteworthy that it is proximity and the need for additional proximity detection devices that has become a potential, if temporary, solution. Furthermore, the focus on proximity, is on the proximity of one device to another, or the implicit proxemic relations that arise from this. Here again, the emphasis is on the embodied characteristics of AR and augmented space in more general. 


\subsection{Margins of the body: augmented space and embodiment}

The site specific nature of augmented space gives rise to another important characteristic and this is the phenomenon of embodiment (Hansen 2004, Massumi 2002 and Allen 2012). It is also a key characteristic that can be applied directly to the understanding of augmented reality. The body itself acts as an interface between the human sensory system and any digital or virtual objects that might be projected into the space inhabited by the user (Fatah, 2016).

Emphasising the embodied characteristics of augmented space and augmented reality would, in addition to proxemic relations, seem central to developing an understanding of participation and engagement, or to be more specific about this, there is a pressing need for the development of a theory that has affective reactions - how the body feels or is moved - that are implicit in the relation of the body to its surroundings. Such an approach would have the fascinating consequence of attaching the body to its surroundings, where the deployment of augmented reality can been see to "expand the body's margin of indetermination" (Hansen, 2004, p. 7). This style of argument has it that the body is at the very centre of interaction, as the "centre of indetermination" (ibid.), the central component in engagement with its surroundings. In sensory or affective terms, the body's relation to the world around it is simultaneously extended and indistinct through forms of augmentation. Thus, technology and, in particular systems such as augmented reality can be seen to be critical tools that can extend the margin between the body and the world (Kirsh, 2013). It is important, therefore, to establish the "framing function" (Hansen, 2004, p. 84 - 87) of the body introduced above, as a characteristic of augmented space, whereby the position and location of the body in space is fundamental to our understanding of the medium and the technology used to deliver virtual objects into real spaces.

The body is always located in real space-time. This is the case even when the body is engaging with content, virtual objects that are generated from outside of the physical location or projected into it. The body in many discourses, modes of representation, and especially those generated across visual, spatial and tactile modalities, is already and always going to be the ultimate frame for information. This property has another important consequence, it "leads to considering how only a blurred distinction seems to exist between space and information, as elements of space increasingly are powerful conveyors of information - materialized into them - becomes more spatially related" (Aurigi, 2008, p. 5). To put this into a more phenomenological context, there is a blurred distinction between the body and the world, and, as in Mark Hansen's terms, we increasingly see "technology as a means of expanding the body's "margin of indetermination" Hansen, 2004, p. 10). Furthermore, that proximity, in the way that it was presented earlier as a distinct issue within augmented space, we see the relation of closeness and distance (Hodge and Kress, 1988) and its corollary, occur as central to the experience of augmented space and in a way that emphasise the affective relations between participants and the media or virtual objects that they interact with. Therefore, one of the most critical questions to come out of this brief discussion of embodiment rests on where to locate the body within augmented space. The body itself acts as an interface both on a sensory level in terms of its reception of 
information from the environment and in terms of how it receives information from devices and displays in an urban environment. Featherstone stresses the "importance of the body as a framer of information and this has become more urgent with digitized media" (Featherstone, 2006, p. 2). In fact, the argument seem to have become even more urgent with the deployment of aspects of augmented reality and its use within real open and urban spaces. The framing function of the body, therefore, is key to a more general understanding of human engagement with a whole range of types of new media and is implicit is the case studies that follow.

\section{Two Case Studies: a descriptive account}

This section provides a descriptive account of two interventions into urban space using aspects of AR and conforming very much into our general understanding of augmented space. Both of the case studies are arenas where both practical strategies The Leytonstone Arts Trail was created as part of a collaboration between The Bartlett, UCL and Holition Ltd entitled "Augmented Urban Reality". It exemplifies many of the characteristics that have been discussed so far in this chapter. First, the trail is an attempt to use augmented reality as part of an integrated set of screen based activities engaged in participants of an arts trail. One important feature is the use of tablets that superimpose an avatar into the space, guide participants through the streets and assist in the finding of objects and other screen based activities that are an integral part of the trail.

One striking feature of this application is the amount of mobility that it affords participants. Positioning of participants has been successful as has the mapping of GPS data to handheld tablets and to virtual objects on screen, the avatar in particular. As such this experience is very much a location based affair and to this effect it most definitely conforms to the characteristics of "site-specificity" as set out the previous section. In addition, in the terms that there are a variety of ways that an experience such as this can be explained using concepts of "embodiment" and framing, not least in terms of the mobility of the body within urban space and the relation set up between the body of the participant, and the virtual actions and reactions of the avatar. Entry points, for example, are a key aspect of the framing of the experience and the proxemic relations that occur as a consequence of this. These case studies are also situations where empirical strategies and specific research agenda can themselves be tested. We ask, to what extent, in the examples that follow, are these interventions located and therefore, site specific. In addition, we need to establish the extent to which the experiences of these space and the interaction with technology and the virtual, are embodied. As argued in the previous section, how are the spatial properties and proxemic relations being managed, whether socially and forms of cultural convention, or through the use of locative technologies such as Bluetooth, GPS or other proprietary technology. A further, leading question is related to how active participation to author AR experiences can be facilitated and to what extent is public led co-creation of AR content used to support agency and thus promote authentic forms of engagement? 


\subsection{Augmented Urban Reality: The Leytonstone Arts Trail}

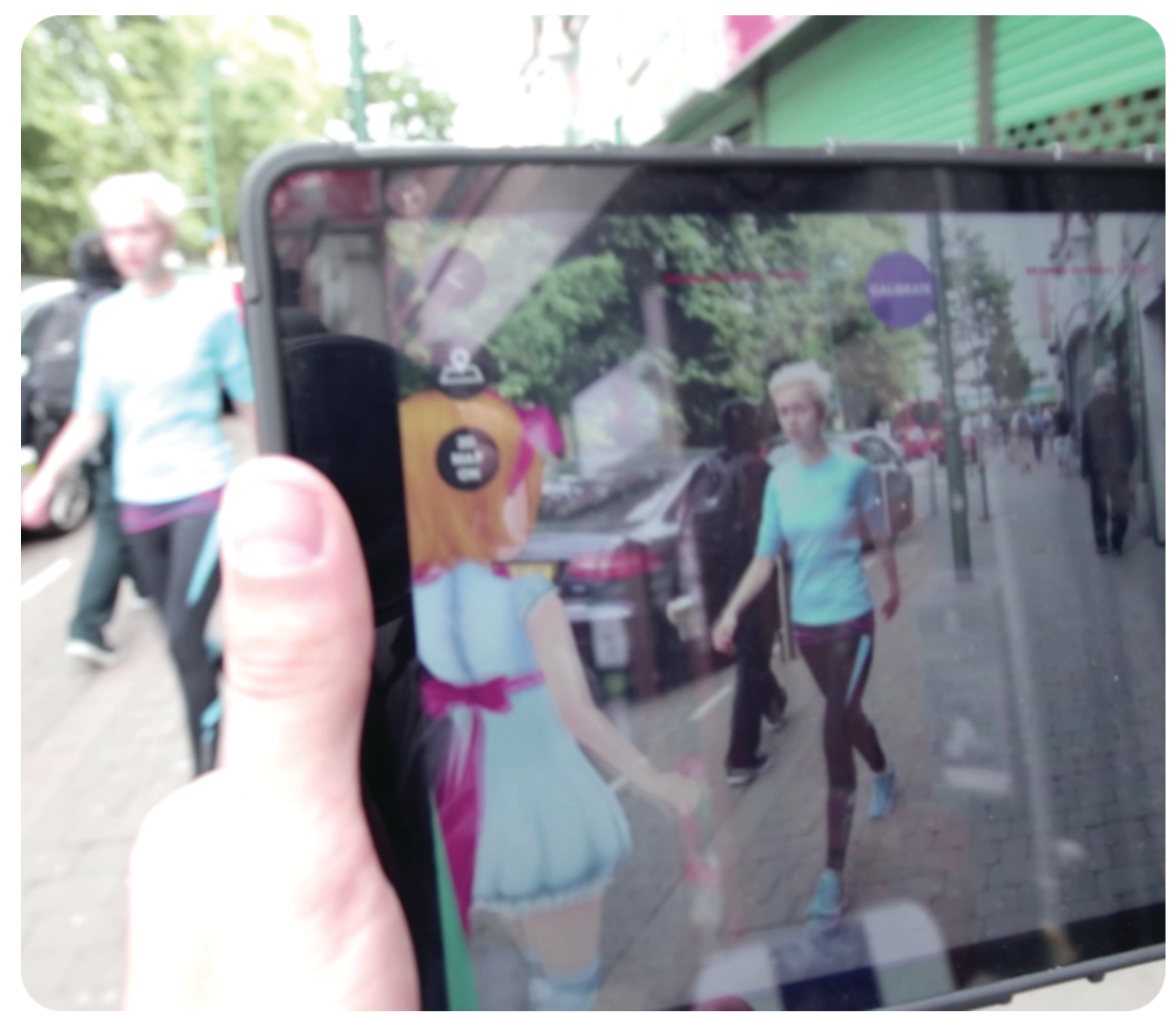

Fig 1. Augmented Urban Space: the Leytonstone Arts Trail, London.

In the AR View one has the choice to enable or disable a 2D map overlay, registered to the 'real' visualized world. The AR view uses a walking $3 \mathrm{D}$ digital character or avatar to guide the user in the exact direction of the selected venue, following the preplanned route. The avatar responds to the body' movements, altering its pose, speed and animation based on the user's movement and proximity to the destination and . The aim is to create new spatial and social narratives and engage people with invisible aspects of their environment through the animated character's movement and behaviour.

During the Leytonstone Arts Trail more than 130 people downloaded the app and used it as a medium to access more content from the event. During 4 days the researchers engaged with passers-by, introduced the project, and helped them installing the app to their devices. Twenty eight questionnaires were gathered from people that were using the app who gave feedback about the different features, such as the User Interface, the AR navigation and sharing pictures with a Live Gallery. A key element here is the potential for people to share aspects of their experience through the live gallery online which also is on display on a situated urban screen on 
the Leytonstone High Street and to generate their own narratives associated with the experience.

Based on feedback received from participants we identified the following aspects:

- Movement and the urban scale: The speed of the digital character influences the user experience and its rhythm. Some users adapted their pace to match that of the digital character. Varying speed could in future be explored, such that the digital character moves and adapts smoothly to the user. Although the digital character is aligned and integrated in the real environment, digital cues could be introduced on the AR view to give a more realistic idea about the exact scale of the digital character and the real distance between both the digital one and the user.

- Hybrid space: As the digital character moves through an urban space it can trigger things of the digital world that are invisible on the real world. Here, it works as a link between the physical and the digital world, and is constantly calling the user's attention to relevant things and information in the environment.

- Multimodal interactions: Some users said it would be interesting to have different animations, audio cues or immediately changing to different modes of display. With regards to the mobile devices used, some tablet users said that they would prefer a lighter, more discreet and portable device like a mobile phone instead of a heavy and attractive tablet, in particular because of handling and safety issues.

- $\quad$ Awareness of the actual environment: This seems to be a very important aspect. Possible risk might not be noticed, such as when crossing a road. The digital character could give a warning or a hint on the screen when getting closer to cross the road.

One key element of this experience is the orientation: contrary to the conventional 2D maps, where a user might feel lost if he or she doesn't know the area or has difficulty orienting, the immersive 3D mediated experience supports natural navigation "it shows me buildings around me... it takes me into a place where I'm very oriented"; "it was a brand new way of navigation to me... there was a restaurant, and without the app I would never thought of entering".

Three crucial moments of interaction were identified during the navigation experience. The first moment is related with initial interaction and engagement with the experience, the point where the user might not know that a digital character will guide him or her. The second moment is during the entire route and the third moment is when the user reaches the destination. In addition, there are important issues around when the user engages with the avatar, further ways of engaging with the avatar could be explored, especially during the first moments of engagement because there are 
times when users started their navigation of the route but were looking in the opposite direction. Entry points are a key element of this and have been explored elsewhere in relation to static $2 \mathrm{D}$ interaction but could easily be applied to real-world 3D interactions. When reorientation of the device is required, there needs to be some system for making the user aware of this at these critical moments and entry points. During the second moment, adding further personalised features to the interaction especially to the avatar, where they might be spoken to directly with personalised data, thus drawing the user's attention to information about the surrounding area and relevant places to visit. Some participants expressed a desire to customise the avatar. The last moments of the trail could be explored with a different animation of the avatar, adding audio or changing to a different mode of display. In addition, many participants expressed a desire to still be able to refer to a 2D map as part of the display and providing an unrestricted view of the surrounding area and much in that same way as with the traditional printed map that was a central part of previous ways of presenting the Arts Trail to participants.

In summary, the approach to AR offered within the Leytonstone Arts Trail offered a digital platform that facilitates various urban encounters and ways that participants could engage with both virtual and real world content. This is part of ongoing work that addresses highlights participant's engagement and strategies for orienting participants to key parts of the experience of an Arts Trail. More generally, as part a longer term research strategy issues of navigation, wayfinding and facilitating meaningful connections between participants and the built environment and how this can be enriched with digitally augmented spatial and social narratives. The argument at this point is that there are many opportunities available, such as the one described in the case study above, whereby AR and augmented space can intensify a participant's sense of place.

\subsection{Cycling Go!}

Cycling Go! combines playing a computer game with riding a bike. If an analogy were needed, imagine Super Mario Kart meets Strava meets Pokémon Go! It may sounds engaging, but perhaps a little dangerous? The point, however, is the opposite, this game is proposed as a means to make cycling safer and to support local sustainable travel initiatives. The spatial, pleasurable sensation you get when riding a bike, even at a moderate, non-risky pace, is already more immersive than playing a sit-down screen-based game, simply because it's real and it is embodied. In addition, the intention to augment cycling in this manner would, in our view, take social gaming to an entirely new level, and would certainly take the current gaming environments indicated by the like of Ingress and Pokemon Go to another level in terms of both user engagement and participation in the outdoors. This is aside from any additional benefit gained from other social and cultural benefits associated from cycling and the development of alternative transport infrastructures. 


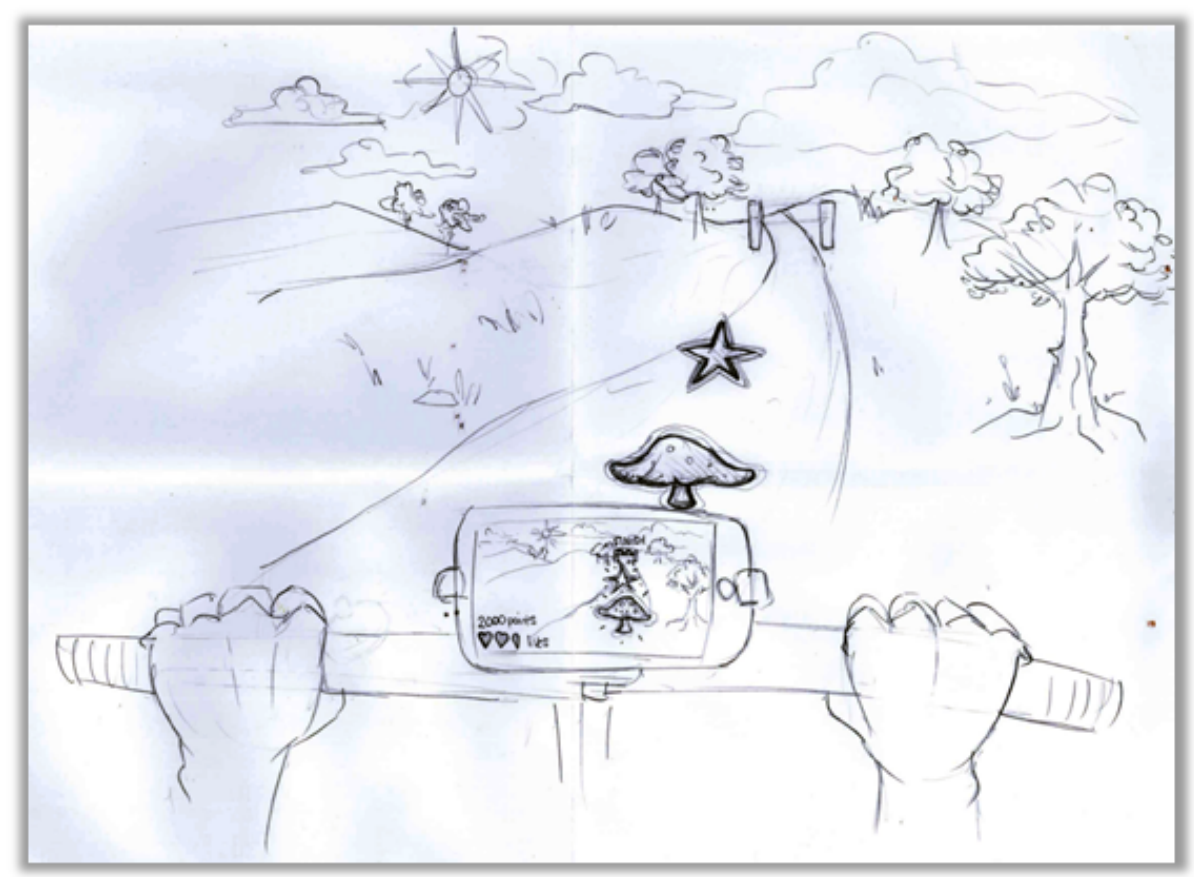

Figure 2: Initial Concept Sketch showing objects that can be 'collected' by the cyclists on offroad route sections, (Drawing courtesy of Tom Martyn).

Cycling Go! specifically aims to increase the take-up of cycling (as an alternative to car travel for distances under five miles) but with a strategic initial focus on usage of key routes, already built at great expense and in the face of challenging

circumstances. These routes have been designated by cycling groups, travel planners, Sustrans and the project's' supporters, CityConnect as key to the Bradford District's 2016-2020 cycling strategy.

A game framework is currently in the planning stages, whereby, content and user administration structures are being created that allows multiple routes, stories and levels (in any city) to be added by others without a high level of technical knowledge. Behind this are two fully working story missions, aimed at key target groups for the take-up of cycling with institutional support from local cycling campaigns groups and the local authority and university (University of Bradford and Bradford Metropolitan District Council) providing a base for user engagement (staff, students and local inhabitants) in addition to the development of targeted research initiatives and infrastructure projects. In this sense design and innovation is quite straightforward as, in the first instance, it 'piggybacks' on both the hype and the more substantive potentials from AR and gamification, such as Pokemon Go! As well as the use of fitness and mapping apps and combines these in order to develop strategies for engagement and the encouragement cycling within urban space.

The major strength of this style approach and the motivation for integrating aspects of AR into this form of engagement with urban space, through the creation of a cycling 
app, is not just about the testing and further development of the core technology, the real strength of this and associated project lies in its ability to engage with its target audience in meaningful ways. Pokemon Go! Could in fact be played on a bike but this was certainly not what the designers had in mind when creating it. Indeed, it actually ceases to function once the rider goes beyond a certain speed (because the current system determines that you are a car) and trying to catch a Pokemon, using two fingers to interact with the screen and aim whilst cycling is frankly very dangerous.

Features of the game in the current prototyping phase are as follows: Adventure story introduction and mission objectives built into journey; Collect virtual items for points (e.g. stars and mushrooms); Position checkpoints on the route (gain points for passing regularly); Upload/add media features for players; Share achievements and play via Facebook and Twitter leaderboards; Team play: compete against another workplace, school, class or group; Unlock cultural interest/engagement points (and visit stops); Safety bonus points and messages; Ghost mode - compete with follow a fellow cyclist or 'cycle ghost' (previous cyclist who has used the route); Link to Strava routes; Report route issues pot-holes and obstacles to local authority; Allow tracking of cyclists by app administrators; Open source add-level functionality available via GitHub and others; Cycling "Treeple" (special game story characters) to interact with enroute.

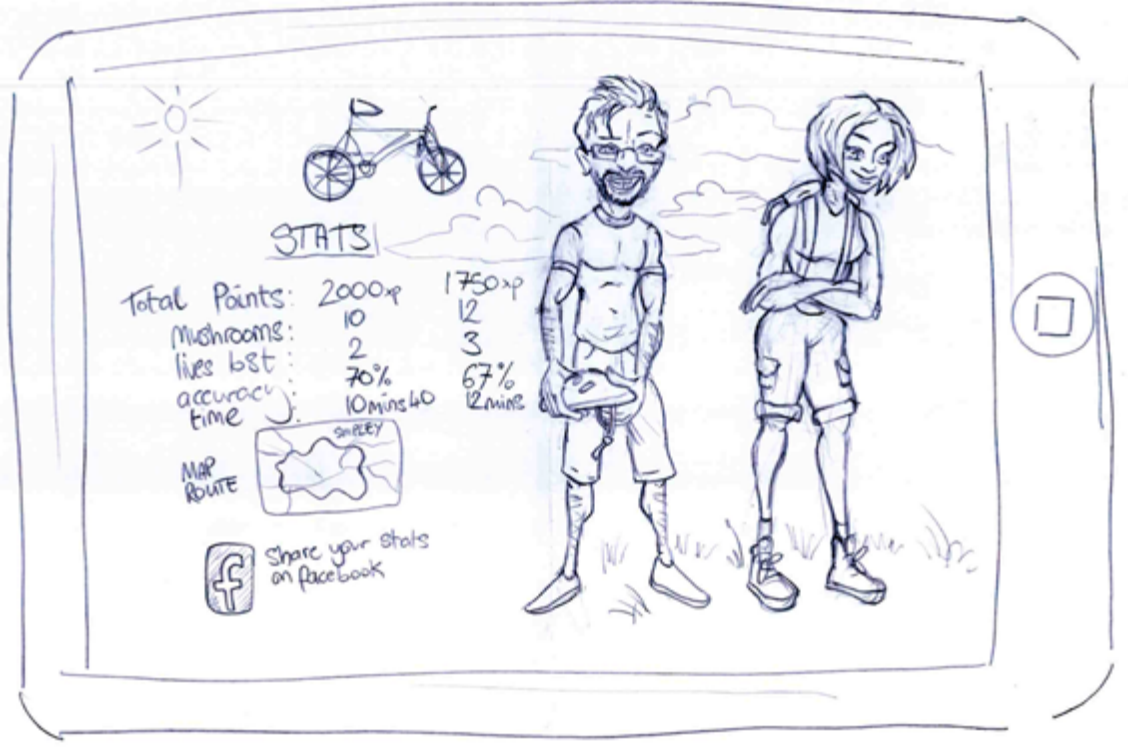

Figure 3: Early Concept Sketch of 'Treeple' Game Characters and Stats., (Drawing courtesy of Tom Martyn).

\section{Conclusion}

There are both technical and social aspects to both of the characteristics of augmented space as discussed earlier and both have been highlighted in the descriptive account given in the case studies. In terms of methodology, a mixed, transdisciplinary 
approach is critical to developing an understanding both the transformative and disruptive potential of these spaces. Such spaces are ultimately, forms of "multimodal text" (Allen, 2008) and the multifaceted nature of these spaces needs to be taken into account at the outset, not just in terms of their potential to accommodate for, or become platforms for the delivery multimodal content, but much more than this. The spaces themselves, as stated, are forms of multimodal text that integrates and combine multimodal properties in and of themselves. This is evidenced, for example, in the need to understand the proxemic relations that occur as a consequence of the application of AR into real public space. Proximity and the spatial as well as social meanings that arise from this most basic characteristic of augmented space is a multimodal principle par excellence. These characteristics, therefore, require a transdisciplinary approach to research from the outset and an approach that prioritises space and its use.

Both case studies have also exemplified important aspects of AR and its impact on the experience of real public space. As a consequence of these initial investigations we continue to advocate a perspective that integrates AR into a wider set of consideration and as part of a more general approach to urban and architectural spaces that have a range of media technologies superimposed onto them. What, for some years now, we have labelled "augmented space". In addition, prioritising the spatial aspects of the design of augmented space has yielded some useful insights into how we are to conceptualise these spaces and not least the need to drive our understanding and the research that informs this by asking the extent to which such spaces are both sitespecific and embodied. To put this another way, an understanding of the variable nature of the space in which augmentation occurs is critical. So too is how the body both relates to the space around it as well as how embodiment, as a set of theoretical concerns, can and should, be used to explain important aspect of interaction and engagement. In addition, and this is most striking, there is an important quality in both AR and augmented space where there is the potential to provide an enhanced a sense of place and this can be achieved by bringing participants to a greater attachment to location rather than dissociating participants from their surroundings as is so often assumed.

Further work in this regard is planned using many of the principles set out in this chapter and, at the time of writing, projects with both regional and global organisations in the museum sector, namely The Bronte Parsonage Museum, West Yorkshire and [ADD: Brighton Museum/s] are being scoped out as part of more general strategies for audience development and public engagement. We have argued that there is a pressing need to go beyond the use of tools most commonly associated with Human Computer Interaction in investigating the use of AR systems in public space. Indeed, some of the methodological distinctions made in this chapter are specifically intended to do this. For example, how can active participation to author AR experiences be facilitated and to what extent is public led co-creation of AR content used to support agency and thus promote authentic forms of engagement? This is where our work will lead us. That is, to a more nuanced and deeper understanding of human engagement with AR systems and augmented space in more general. 


\section{Reference List}

Alias (2004) “A semiotic study of Singapore's Orchard Road and Marriot Hotel”, in O’Halleran (ed.) Multimodal Discourse Analysis. Continuum.

Allen, P. (2008): "Framing, Navigation and the Body in Augmented Public Spaces", Augmented Urban Spaces, Aurigi (ed.), Ashgate Press, London.

Allen, P. (2009) "Place and Locality in Augmented Public Space": a case study on the site-specific nature of urban screens, Proceedings of the IEEE, Cyberworlds 20, E09.

Allen, P. (2012) "Framing the Media Architectural Body". Proceedings of the $A C M$, Media Architecture Biennale, Aarhus, Denmark 2012.

Aurigi, A. ed. (2006) Augmented Urban Spaces. Ashgate Press, London.

Azuma, R. (1997) “A Survey of Augmented Reality”, in Presence: Teleoperators and Virtual Environments, 6, 4 (August 1997).

Fatah, A Penn, A and O’Neill, E (2008) "Mapping, sensing and visualising the digital co-presence in the public arena" in Timmermans, H and de, VB, (eds.) Design \& Decision Support Systems in Architecture and Urban Planning. (pp. 38-58). TU/e: Leende, The Netherlands.

Fatah, A. (2009) "Towards an Integrated Architectural Media Space: The Urban Screen as a Socialising Platform", in Urban Screens Reader, eds. McQuire, S, Martin, $\mathrm{M}$ and Niederer, S, Institute of Network Cultures, Amsterdam.

Fatah gen. Schieck, A, O’Neill, E and Kataras, P (2010) "Exploring Embodied Mediated Performative Interactions in Urban Space", UbiComp'10, September 26-29, 2010, Copenhagen, Denmark. ACM 978-1-60558-843-8/10/09.

Fatah gen. Schieck, A. (2016). Living Architecture: Currencies between Architectural Project Pedagogy and Time-based Media Performance. aae 2016 Research Based Education. Bartlett School of Architecture.

Featherstone, M. (2006) Body Image/Body without image, in Theory, Culture and Society, number 23, SAGE.

Gartner (2016) 3 Trends appear in the Gartner hype cycle of emerging technologies, 2016.

http://www.gartner.com/smarterwithgartner/3-trends-appear-in-the-gartner-hypecycle-for-emerging-technologies-2016/ - accessed 20/03/17.

Hall, E. (1966) The Hidden Dimension, Random House, US.

Hansen, M (2004) New Philosophy for New Media, MIT Press. 
Hodge, R. and Kress, G. (1988) Social Semiotics. Polity, Cambridge, UK.

Humphery-Jenner, M. (2016) "What went wrong with Pokemon Go? Three lessons from plummeting numbers", https://theconversation.com/what-went-wrong-withpokemon-go-three-lessons-from-its-plummeting-player-numbers-67135 - accessed $18 / 10 / 16$

Julier, S., Fatah gen. Schieck, A., Blume, P., Moutinho, A., Koutsolampros, P., Javornik, A., Kostopoulou, E. (2016). VisAge: Augmented Reality for Heritage. PerDis 2015, Oulu, Finland.

Kirsh, D. (2013 Embodied cognition and the magical future of interaction design. ACM Trans. Computer-Human Interaction 20 (1)

Massumi, B. (2002) Parables of the Virtual, Duke University Press, Durham and London.

Kwon, M. (2002) One Place After Another: Site-Specific Art and Locational Identity, MIT Press.

Manovich, L. (2006) The Poetics of Augmented Space, Visual Communication Journal, SAGE, vol 5(2): 219-240.

McCarthy, A. (2003) Ambient Television: visual culture and public space. Duke University Press, Durham and London.

O'Toole, M (2004) "Opera Ludentes: the Sydney Opera House at work and play" in O’Halleran (ed.) Multimodal Discourse Analysis. Continuum.

Robison, D. (2012) "Learning on Location with Ami: The Potentials and Dangers of Mobile Gaming for Language Learning", in Left to My Own Devices: Learner Autonomy and Mobile-Assisted Language Learning (pp 67-88). BRILL.

Rogers, Y. 2011. Interaction Design gone Wild: Striving for Wild Theory, Interactions, 18(4), pp. 58-62.

Tassi, P. (2016) "What Mystery 'Pokémon GO' Device Is Nintendo Making Now?", https://www.forbes.com/sites/insertcoin/2017/02/03/what-mystery-pokemon-godevice-is-nintendo-making-now/\#4840872a15ea - accessed 03/02/17.

The Hollywood Reporter (2016) http://www.hollywoodreporter.com/lists/pokemongo-guide-game-tips-real-life-hazards-910304/item/players-lured-by-armed-robbers910332 - accessed 07/12/16. 